Research Paper:

\title{
Correlation Between Health Literacy and Self-efficacy of the Elderly
}

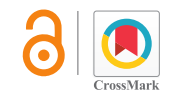

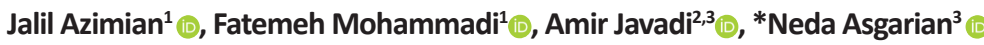

1. Department of Nursing, School of Nursing and Midwifery, Qazvin University of Medical Sciences, Qazvin, Iran. 2. School of Medicine Social Affairs, Tehran University of Medical Sciences, Tehran, Iran.

3. Department of Statistics and Informatics, School of Medicine, Qazvin University of Medical Sciences, Qazvin, Iran.

\begin{tabular}{|c|c|}
\hline $\begin{array}{l}\text { Use your device to scan } \\
\text { and read the article online }\end{array}$ & \\
\hline 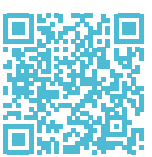 & $\begin{array}{l}\text { Citation Azimian J, Mohammadi F, Javadi A, Asgarian N. [Correlation Between Health Literacy and Self-efficacy of the Elderly (Per- } \\
\text { sian)]. Journal of Inflammatory Diseases. 2020; 24(1):224-233. https://doi.org/10.32598/JQUMS.23.5.3 } \\
\text { dol' https://doi.org/10.32598/JQUMS.23.5.3 }\end{array}$ \\
\hline
\end{tabular}

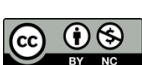

Received: 08 Oct 2018 Accepted: 21 Jan 2019 Available Online: 01 Apr 2020

Keywords: Health literacy, Selfefficacy, Elderly

\section{A B STRACT}

Background Health literacy is the capacity of a person to obtain, process, and understand basic health information for appropriate health decision making. Self-efficacy is the assurance that is felt about a particular activity which maintains and promotes effective health behaviors.

Objective The purpose of this study is to evaluate the relationship between health literacy and self-efficacy in the elderly people.

Methods This descriptive-correlational study with cross-sectional design was conducted on 144 elderly living in Ramsar city in 2018 who were selected using a random cluster sampling method. Data collection tools were a demographic form, Self Rated Abilities for Health Practices Scale, and Health Literacy for Iranian Adults completed by the researcher after obtaining informed consent from the participants. Data were analyzed in SPSS v.16 software using correlation test, independent t-test, chi-square test, and Fisher's exact test.

Findings Most of participants were female (59\%), married (86.8\%), housekeeper (52.8\%) and high school dropouts (35.4\%), and moderate financial status (61.8\%) with a mean age of $68.75 \pm 2.12$ years. Pearson correlation test results showed a significant relationship between health literacy and self-efficacy of the elderly ( $\mathrm{P}=0.0001)$.

Conclusion Considering the role of health literacy in increasing the self-efficacy of the elderly, healthcare system policymakers need to pay attention to the issue of health literacy in health system planning.

\section{Extended Abstract}

\section{Introduction}

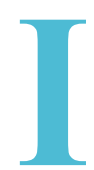

$\mathrm{n}$ the last hundred years, the average life expectancy in developed countries has increased by about 30 years and has reached 80 years [1]. The United Nations estimates that the world's elderly population will increase to 2.1 billion by 2050 [2]. More than $9.3 \%$ of Iran's population is over 60 years old [3]. Access to or awareness of information related to health issues is one of the most important factors in determining the health of the elderly. For this purpose, the elderly need a high level of health literacy. Health literacy is defined as a person's ability to obtain, process, and understand basic health information for making the right health decision [6].

\section{* Corresponding Author:}

Neda Asgarian

Address: Department of Statistics and Informatics, School of Medicine, Qazvin University of Medical Sciences, Qazvin, Iran.

Tel: +98 (11) 55222031

E-Mail: n.asgarian1366@gmail.com 
Adaptive and health-promoting behaviors in the elderly, such as high health literacy, have a potential impact on health promotion, increasing self-efficacy and quality of life, as well as reducing health care costs [9]. Self-efficacy in all age groups, especially in the elderly, has been widely discussed among experts in terms of their age-related and physiological changes and vulnerability. Self-efficacy is a person's confidence in ability to perform a particular behavior in a specific situation that can lead to desired outcomes [10].

The association of declining health literacy with the increase of age is now considered an important challenge and risk in health care systems [11]. Elderly people with optimal health literacy will see a doctor in a timely manner in accordance with the issues, problems and diseases when they have not yet led to disability; they learn to take action before they become incapacitated, learn prevention methods, become familiar with the sources of support, and as a result, have maximum efficiency of their minimum capabilities [12]. This study aimed to investigate the relationship of health literacy with self-efficacy in the elderly people.

\section{Materials and Methods}

This descriptive-correlational study with cross-sectional design was conducted on 144 elderly living in Ramsar city in 2018 who were selected using a random cluster sampling method. Data collection tools were: 1 . A demographic form surveying age, gender, marital status, level of education, occupation, income level, having knowledge about health literacy, and having the disease and its type; 2. Self Rated Abilities for Health Practices Scale developed by Becker et al. [13] whose Persian version has been evaluated and its acceptable validity and reliability have been reported by Azadbakht et al. [11] and Pour vakhshoori et al. [12]; and 3. Health Literacy for Iranian Adults Questionnaire which has good validity and reliability according to Montazeri et al. [14].

\section{Results}

The majority of seniors (59\%) have low health literacy; $43.5 \%$ of them had moderate self-efficacy and $41 \%$ good self-efficacy. According to Table 1, the Pearson correlation test results showed a positive and significant relationship between health literacy and self-efficacy of the elderly ( $\mathrm{r}=$ 0.71, $\mathrm{P}=0.0001$ ).

\section{Discussion}

The results of the present study showed that the most of the elderly (59\%) had low health literacy. This is consistent with the results of Reisi et al. [16]. In their study, the level of health literacy was $8.8 \%$, which indicates that it is insufficient in people over 60 years of age. Our results are also in agreement with the findings of Hosieni et al. [17]. Most seniors had moderate to good self-efficacy. In the studies by Baljani et al. and Naseh et al., the elderly had low general self-efficacy $[18,19]$. According to the results of the present study, it can be said that the higher the health literacy, the higher the self-efficacy; which is consistent with the results of Osborn et al. and Rafiezadeh et al. [20, 23].

Given the important role of health literacy in increasing the self-efficacy of the elderly and subsequently increasing active and independent aging in such a way that they will not be dependent on children and the government, improvement of health literacy reduces the financial burden of care for the elderly and leads to active aging community and skilled and experienced workforce. National policies need to be put on the agenda to address the issue of health literacy and related educational programs. In order to increase the awareness of the elderly in the field of health literacy and self-efficacy, it is possible to develop an educational protocol at the national level on the importance of health literacy and provide the required equipment to facilitate it.

Table 1. Correlation of health literacy and self-efficacy in the elderly

\begin{tabular}{ccc}
\hline Variables & Health Literacy & Self-efficacy \\
Health literacy & 1 & 0.714 \\
Pearson correlation coefficient (R) & - & $000 / 0$ \\
$\mathrm{~N}$ & 144 & 142 \\
Self-efficacy & 0.714 & 0.000 \\
Pearson correlation coefficient (R) & & 1 \\
$\mathrm{~N}$ & 142 & 142 \\
\hline
\end{tabular}


(Previous Title: The Journal of Qazvin University of Medical Sciences)

\section{Ethical Considerations}

Compliance with ethical guidelines

This study was approved by the Ethics Committee of Qazvin University of Medical Sciences (Code: IR.QUMS. REC.1396.180).

Funding

This study was extracted from the MSc. thesis of forth author Department of Statistics and Informatics, School of Medicine, Qazvin University of Medical Sciences.

Authors' contributions

All authors contributed in preparing this article.

Conflicts of interest

The authors declared no conflict of interest. 


\title{
بررسى همبستخى سواد سلامت و خودكار آمدى سالمندان شهرستان رامسر در سال جوس
}

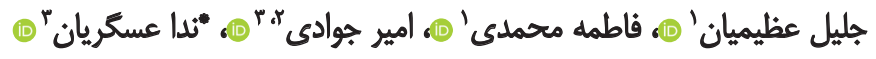

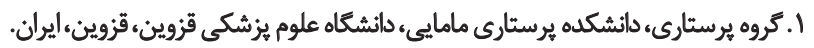

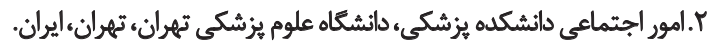

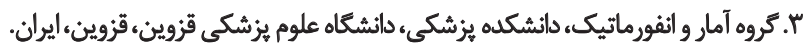

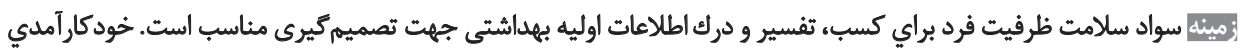

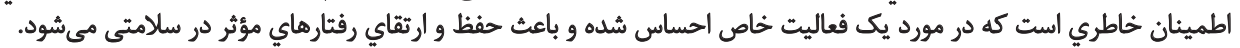

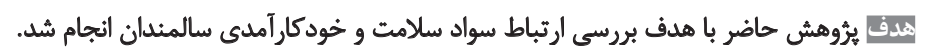

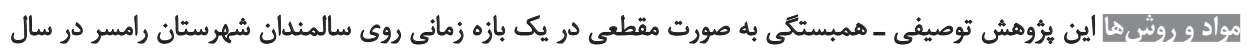

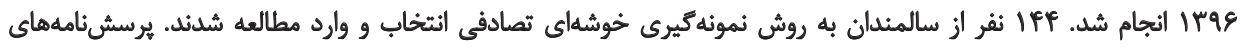

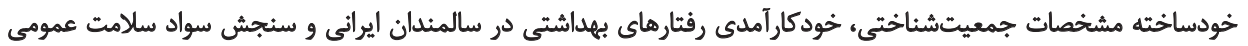

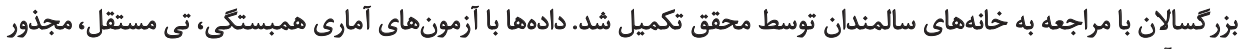
كاي و آزمون دقيق فيشر تحليل شيدند.

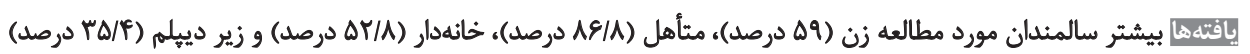

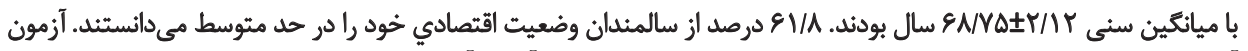

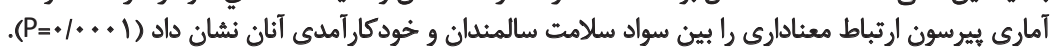

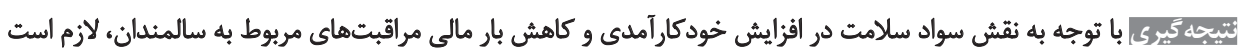

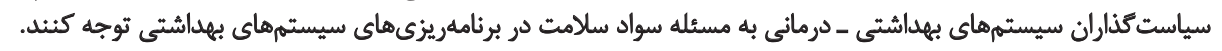
تاريخ انتشار: " آ فروردين

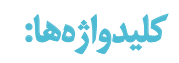

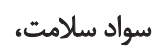
خودكار آمدى، سالمندان
افزايش ناتواني است؛ درنتيجه سالمندي به يك معنى افزايش

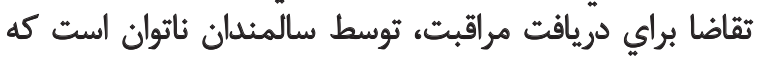

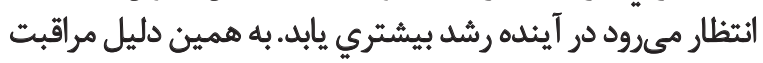

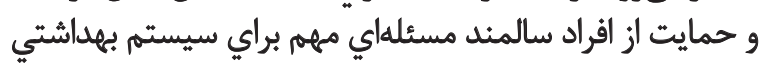

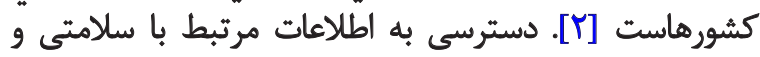

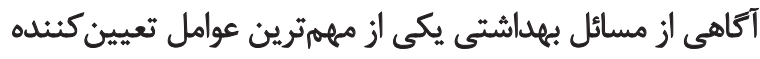

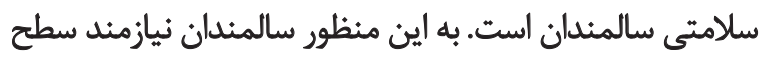
بالايى ازٔ سواد سلامت هستيند.

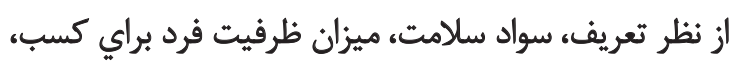

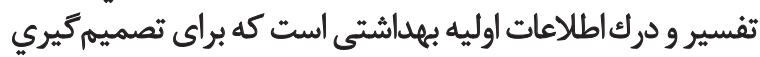

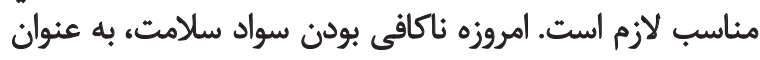

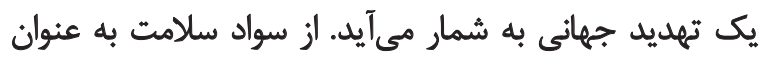

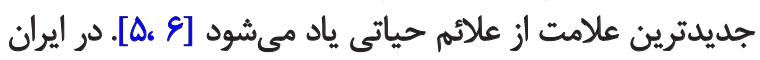

dale در صد سال كذشته در كشور هاى بيشرفته ميانگين عمر انسان

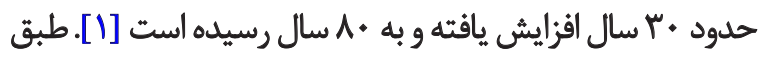

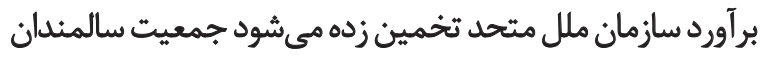

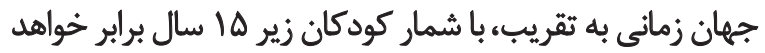

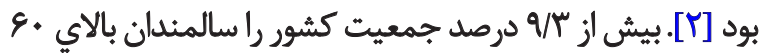

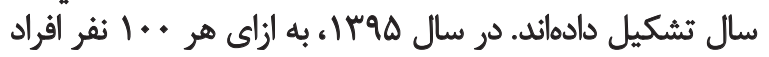

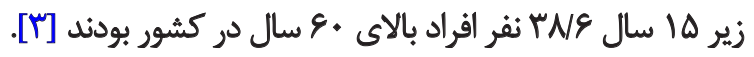

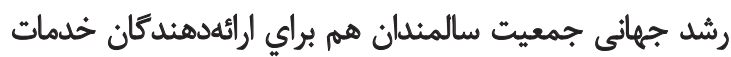

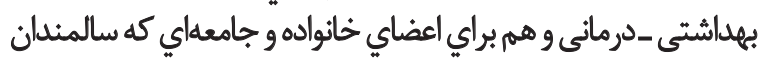

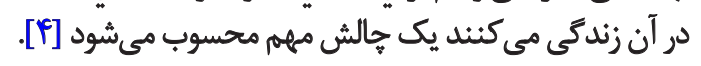
از جالشهاي مهم افزايش جمعيت سالمند در جوامع امروز، 


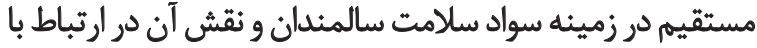

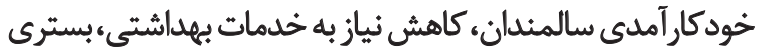

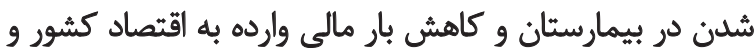

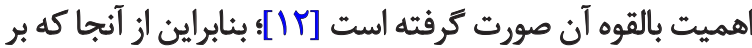

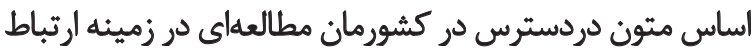

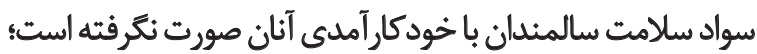

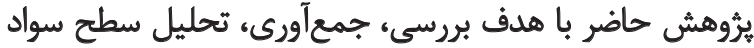

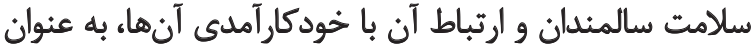
مرحله زيربنايى فرايند آموزش انجام شد اندا

\section{مواد و روشها}

اين يُروهش با رويكرد كتّى به صورت توصيفى ـ همبستكى

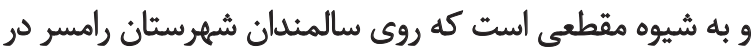

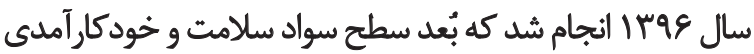

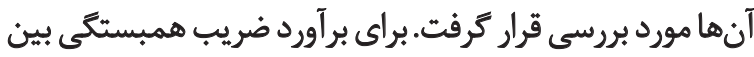

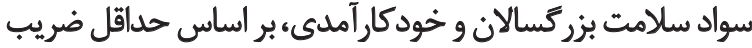

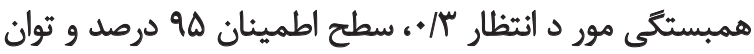

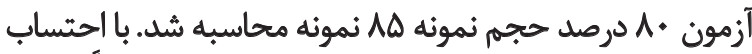

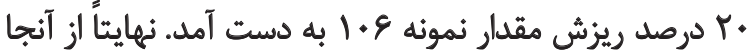

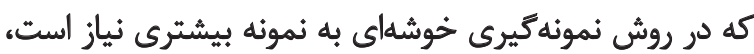

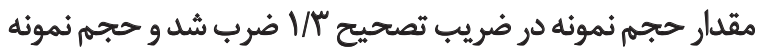

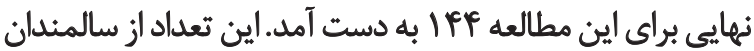

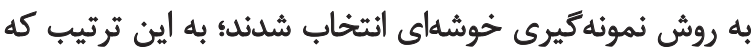

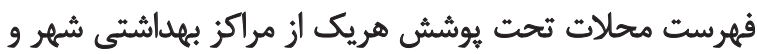

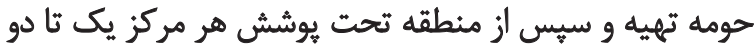

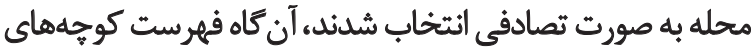

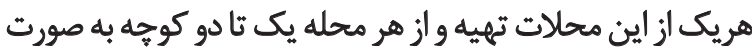

$$
\text { تصادفى ساده ائتخاب شد. }
$$

به ساكنين خانههاي اين كوجهها مراجعه شد و در صورت

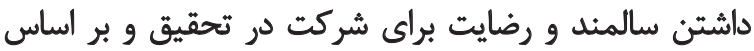

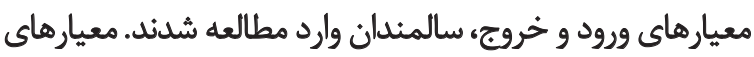

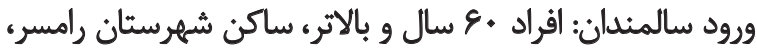

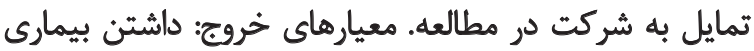

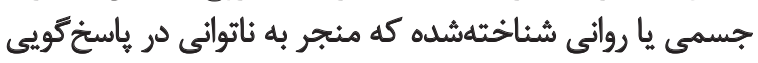

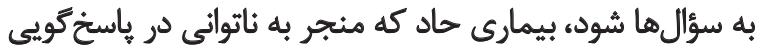

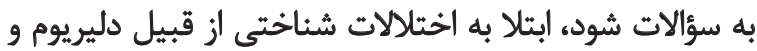

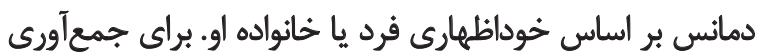

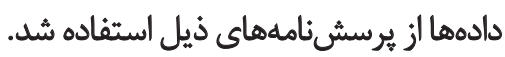

يرسشنامه خودساخته مشخصات جمعيتشناختى كه شامل

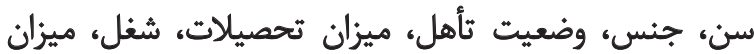

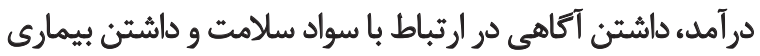

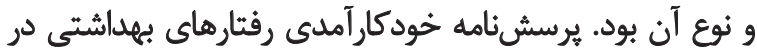

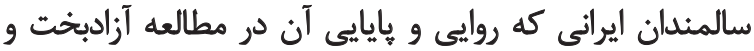

نيز بررسى متون، نشاندهنده سواد سلامت مرزى تا ناكافى در

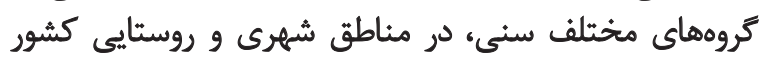

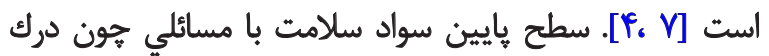

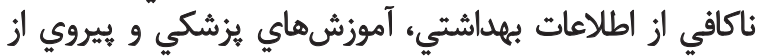

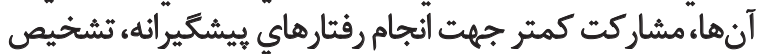

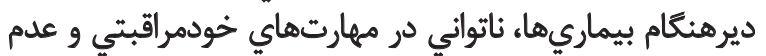
تبعيت از رفتارهاي سبك زنديمي سالهم مرتبط است.

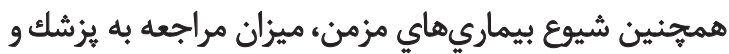

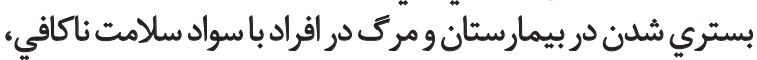

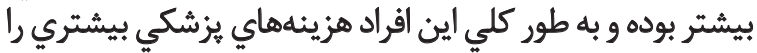

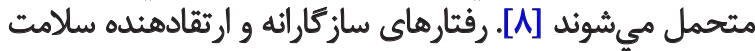

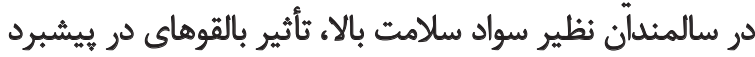

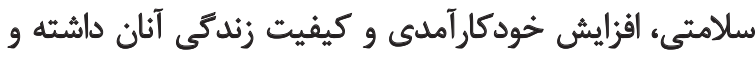

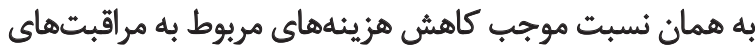

بهداشتى خواهد شد [9].

خودكارآمدي در تمام دورههاى سني بلويرّه در سالمندان

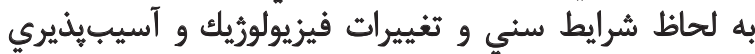

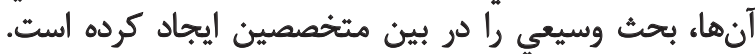

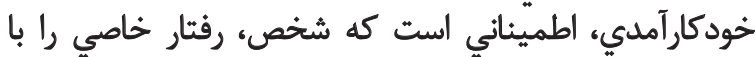

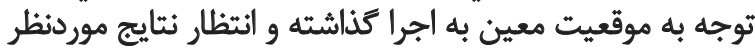

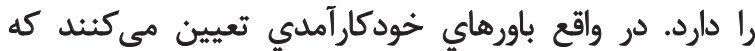

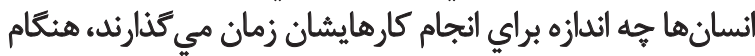

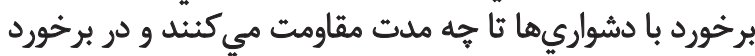

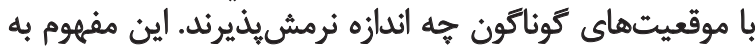

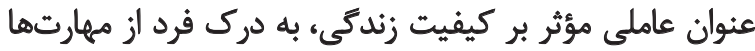

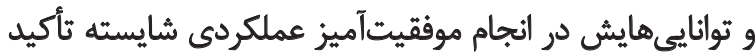

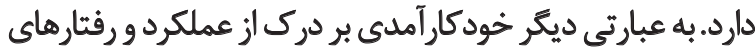

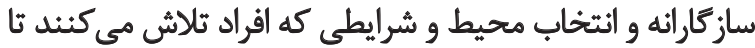
به آن دست يابند، اثر مى كذارد [ • [1].

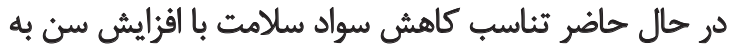

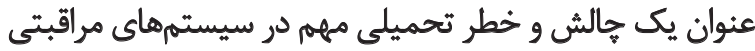

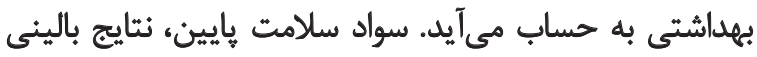

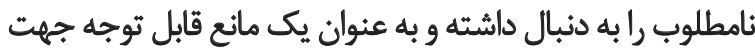

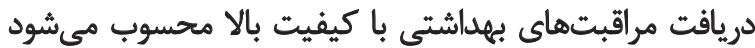

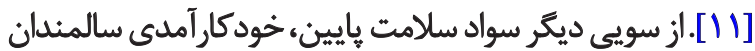

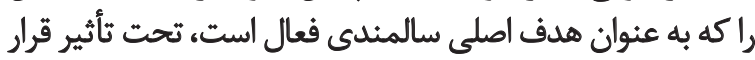

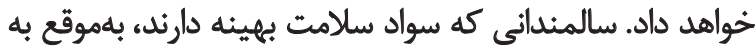

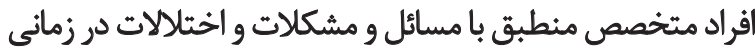

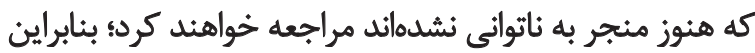

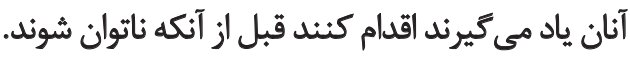
روشهاى بيشغيرى را مى آموزند، منابع حمايتى را خواهند

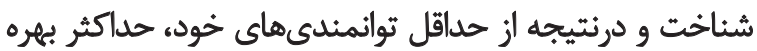
را خواهند برد. علاوه بر اين، مطالعات بسيار اندكى بوني بهائ طور بهره 


\section{يافتهان}

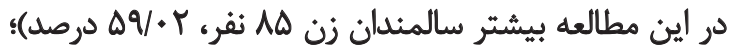

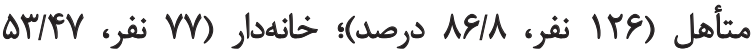

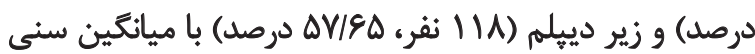

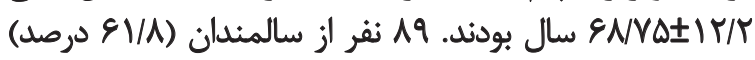

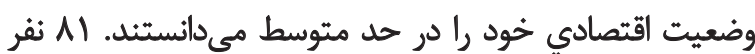

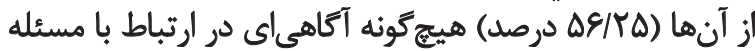

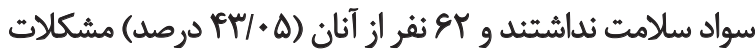

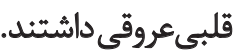

طبق جدول شماره ا، شيوع سواد سلامت ناكافي در جمعيت

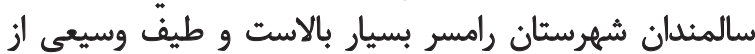

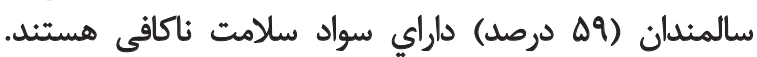

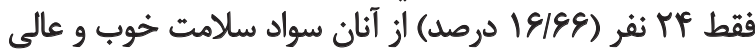

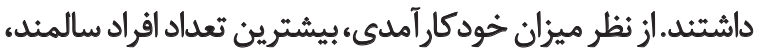

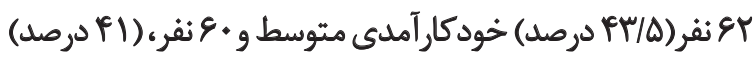

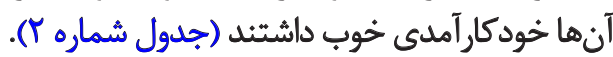

طبق يافتههاى يثروهش حاضر، آزمون آمارى فيشر ارتباط

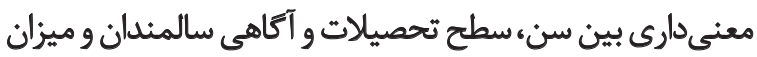

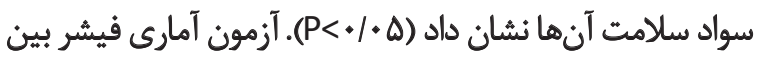

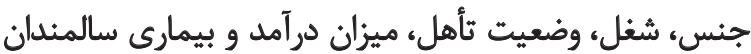

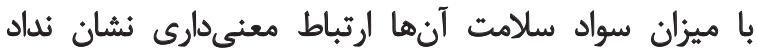

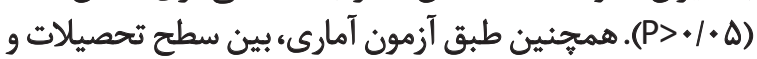

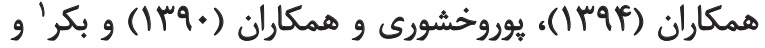

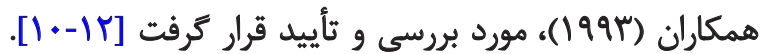

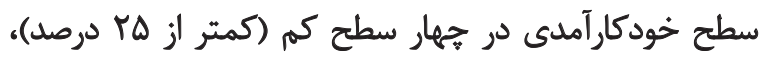

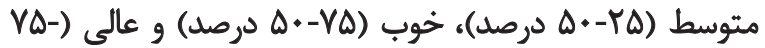

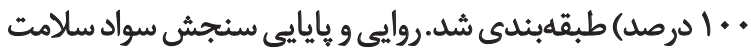

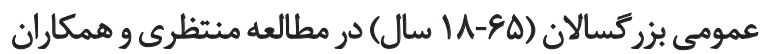

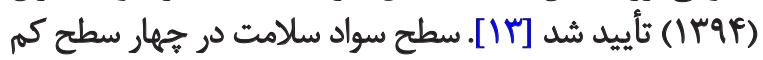

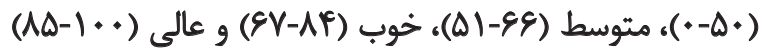

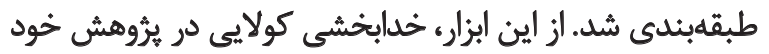

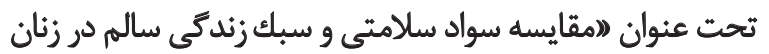

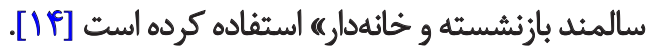

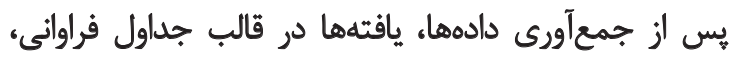

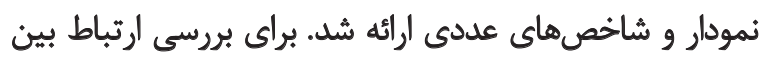

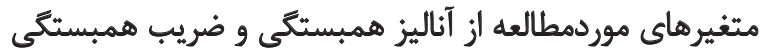

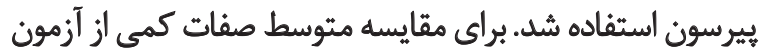

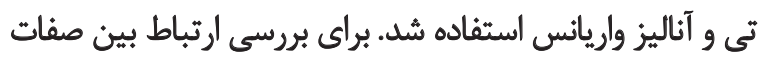

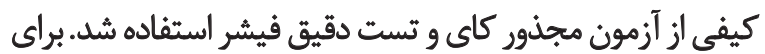

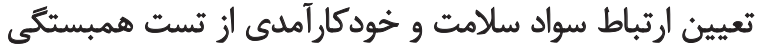

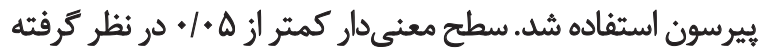

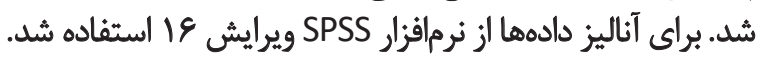

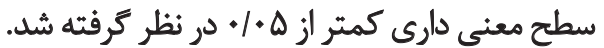

\section{Becker}

جدول ا. توزيع فراوانى ميزان سواد سلامت سالمندان

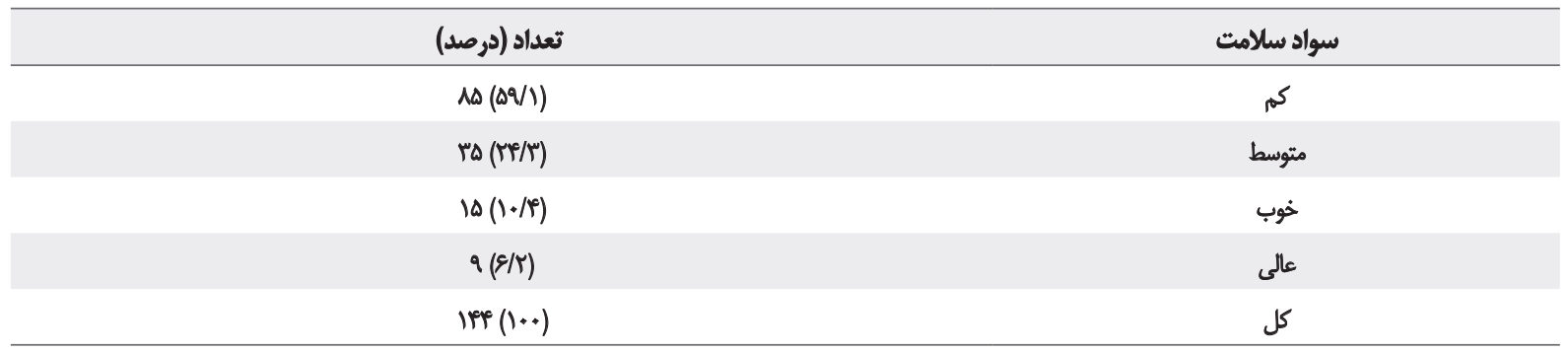
بيمـالكهــاى التــهابى

جدول ب. توزيع فراواني ميزان خود كار آمدى افراد سالمثد

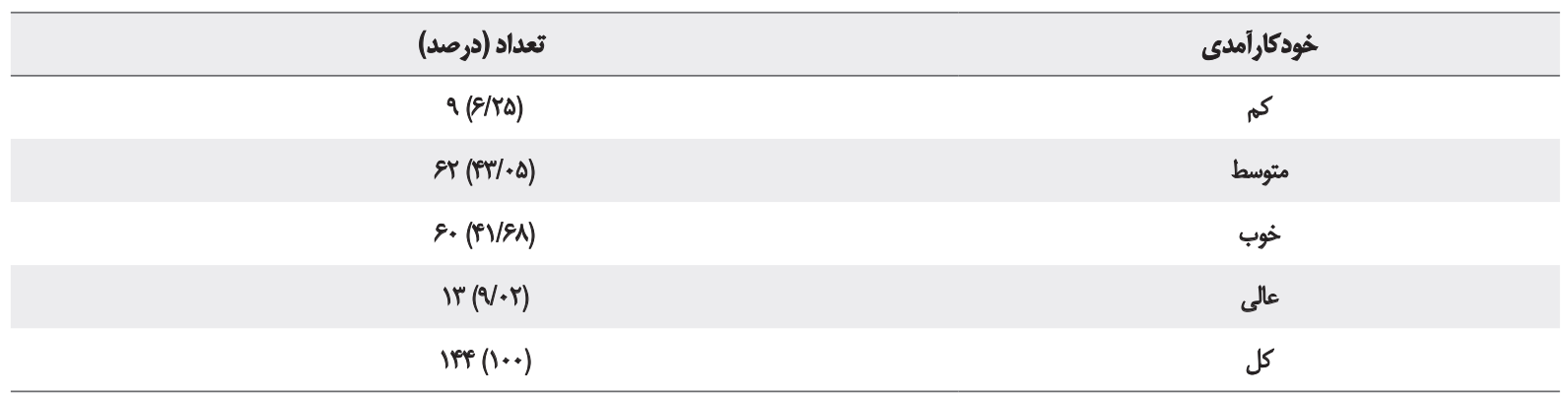

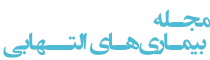


جدول "ا. همبستكى سواد سلامت و خودكار آمدى سالمندان

\begin{tabular}{|c|c|c|}
\hline \multicolumn{3}{|c|}{ همبستكي سواد سلامث وخودكار آمدى سالمندان } \\
\hline حودكار آمدى سالمندان & سواد سلامت سالمندان & 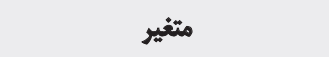 \\
\hline.$M i f$ & 1 & سواد سلامت سالمنلان \\
\hline \multicolumn{2}{|c|}{$\%$} & همبستكى ييرسون در سطح معنادارى \\
\hline ine & inf & تعلداد \\
\hline 1 &.$M I F$ & خودكارآمدى سالمندان \\
\hline \multicolumn{2}{|c|}{$\%$} & همبستكى يرسون در سطح معنادارى \\
\hline Iff & Iffe & تعلتاد \\
\hline
\end{tabular}

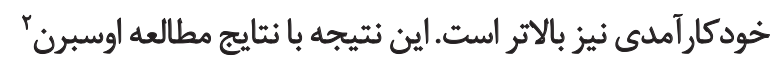

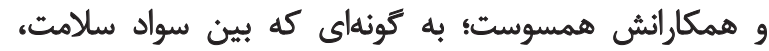

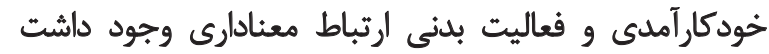

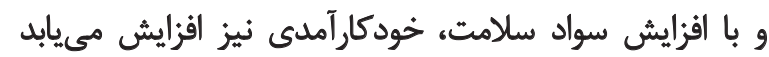

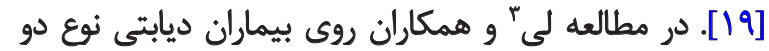

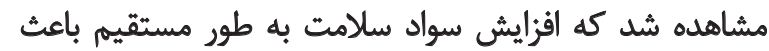

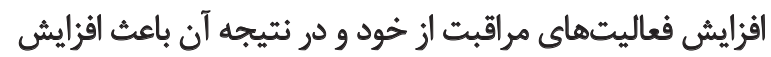

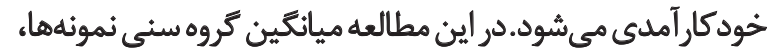

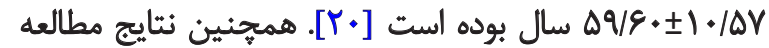

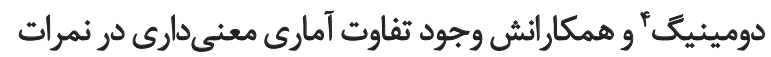

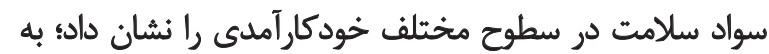

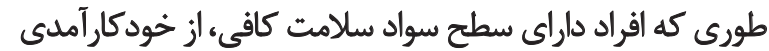

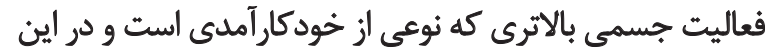

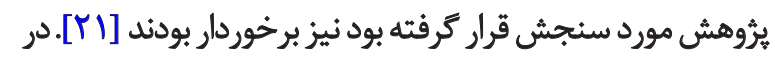

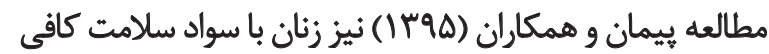

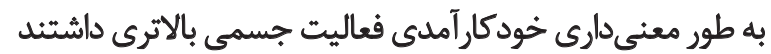

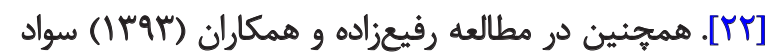

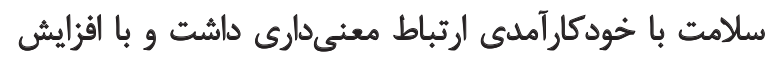

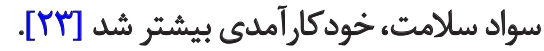

نتايج مطالعه حاضر مي تواند يايه و زميينهاي براى برؤوهشي هاي

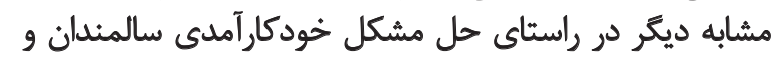

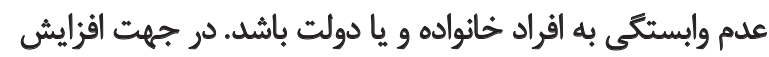

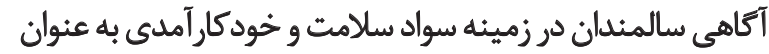

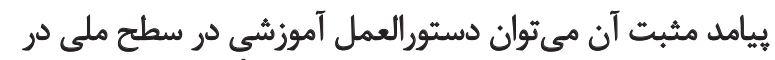

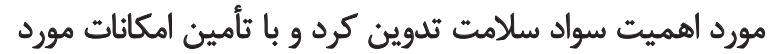
نياز سهولت امر را ثأمين كرد.

قابليتهاى سواد سلامت شامل درك سازماندهى اطلاعات،

2. OSBERN

3. LEE

4. DOMINIK

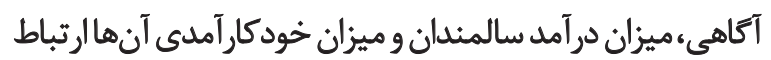

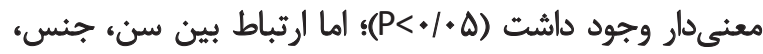

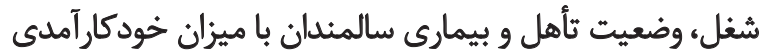

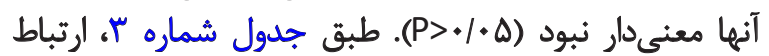

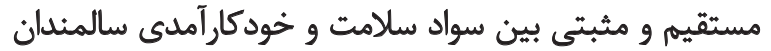

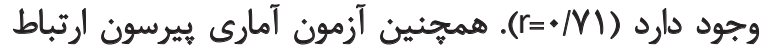
معنى دارى را بين سطح سواد سلامت و خود كارآمدى سالمندان

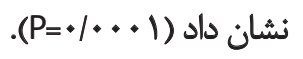

\section{s.sdowis}

هن نوايج مطالعه حاضر نشان داد طيف وسيعى از سالمندان

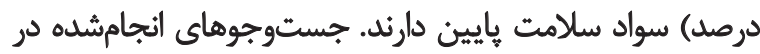

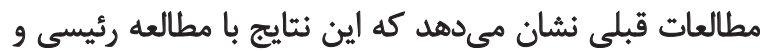

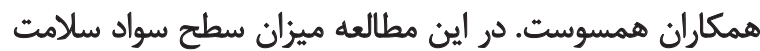

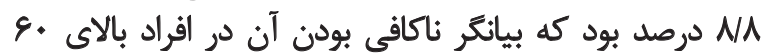

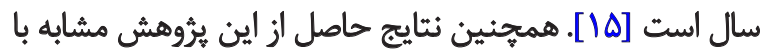

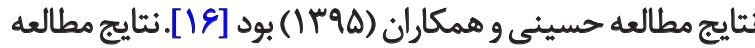
حاضر نشان داد كه بيشتر سالمندان خود كارآمدى در حد حد متوسط

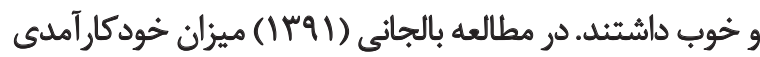

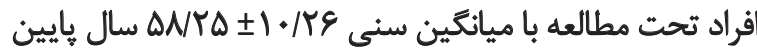

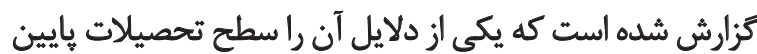

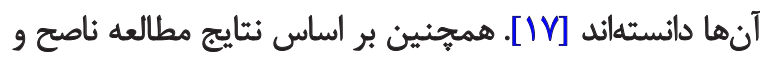

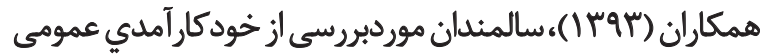

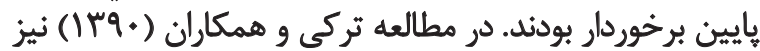

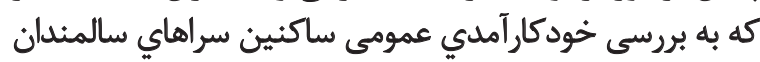

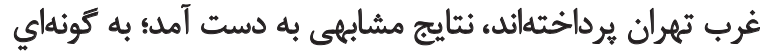

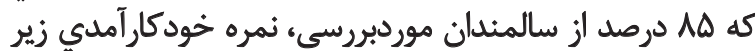

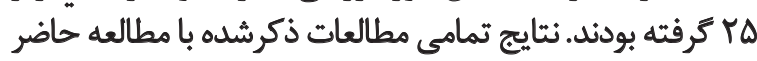

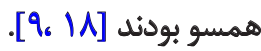
طبق نتايج بُروهش حاضر هرجه سواد سلامت بالاتر باشد؛ 
توانايى جستوجو در ميان انواع ابزارهاى جايى و الكترونيكي

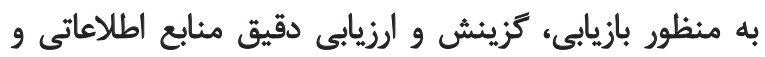

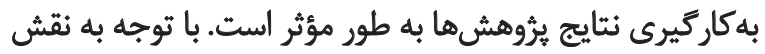

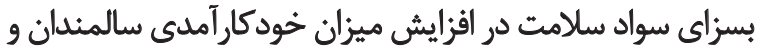

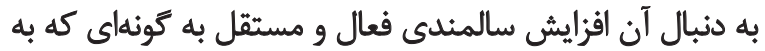

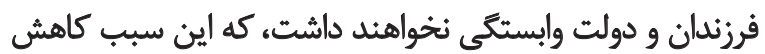

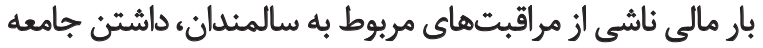

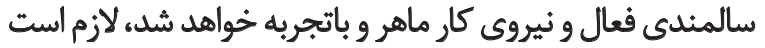

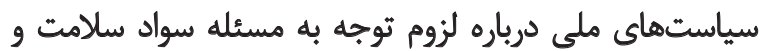

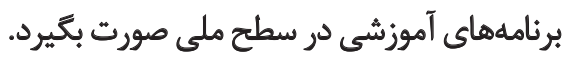
مالاحناث أخالاقي يميروى از اصول اخلاق يثوهش اين مطالعه با كد IR.QUMS.REC.1396.180 مورد تأييد كميته اخلاق دانشكاه علوميزشكى قزوين قرار ترفت.

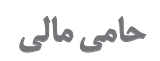

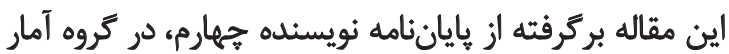

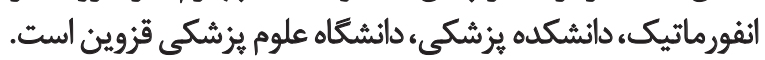

$$
\text { مشاركت نويسيند مَان }
$$

تمامى نويسندكان در نكارش اين مقاله مشاركت داشتهاند.

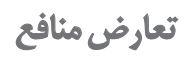

بنا به اظهار نويسندكان اين مقاله تعارض منافع ندارد. 


\section{References}

[1] Torkani S. Older adults; Self- efficacy \& nursing. Tehran: Jameehnegar; 2015. [In Persian] http://opac.nlai.ir/opac-prod/ bibliographic/3937490

[2] United Nations Information Centre (no author) The elderly population of the world [online]. 2016 Available from: http://www.unicir. org/index.php?option=com_content\&view=article\&id=2254

[3] Memarian R. Application of nursing concepts and theories. Tehran: Heidari; 2013. [In Persian] http://opac.nlai.ir/opacprod/bibliographic/3226640

[4] Abdolahi A. Aging health. Tehran: Jameenegar; 2013. [In Persian] http://opac.nlai.ir/opac-prod/bibliographic/3106898

[5] Secretarial National Council of the Elderly (no author) the elderly population of the Iran [online]. 2016 Available from: http://www.snce.ir/435454354-3/

[6] Mohseni M, Khanjani N, Iranpour A, Tabe R, Borhaninejad V. The relationship between health literacy and health status among elderly people in Kerman. SALMAND. 2016; 10(2):149-58. [In Persian] http://salmandj.uswr.ac.ir/article-1-790-en.html

[7] Noroozian M. The elderly population in Iran: An ever growing concern in the health system. Iran J Psychiatry Behav Sci. 2012; 6(2):1-6. [PMID] [PMCID]

[8] Khatti Dizabadi F, Yazdani J, Eftekhar Ardebili H, Batebi A, Shojaezadeh $D$. the status of caregiving among informal caregivers of community-dwelling elderly. J Mazandaran Univ Med Sci. 2013; 23(100):31-41. [In Persian] http://jmums.mazums.ac.ir/ article-1-2117-fa.html.

[9] Torki Y, Hajikazemi E, Bastani F, Haghani H. General self efficacy in elderly living in rest-homes. Iran J Nurs. 2011; 24(73):55-62. [In Persian] http://ijn.iums.ac.ir/article-1-1046-en.html

[10] Azadbakht M, Taheri Tanjani P, Garmaroudi Gh, Sahaf R, Shojaee $D$. Validity and reliability of self efficacy of health practice scale (SRAHPS) in Iranian elderly. J Sabzevar Uni Med Sci. 2015; 22(3):490-8. [In Persian] https://www.magiran.com/ paper/1414358/?lang=en

[11] Pour Vakhshoori N, Pasha A, Ghanbari A, Atrkar Roshan Z. Relationship between Self-efficacy and Health Behaviors of Faculty Members of Guilan University of Medical Science. Iran J Nurs. 2011; 24(70):39-48. [In Persian] http://ijn.iums.ac.ir/ article-1-984-en.htm

[12] Becker $\mathrm{H}$, Stuifbergen A, OH HS, Hall S. Self-rated abilities for health practices: A health self-efficacy measure. J Health Behav Educ Promot. 1993; 17(5):42-50. https://psycnet.apa.org/ record/1994-08112-001

[13] Montazeri A, Rakhshani F, Azin A, Jahangiri K, Ebadi M, Naderimagham Sh, Health Literacy for Iranian Adults (HELIA): development and psychometric properties. Payesh. 2014; 13(5):58999. [In Persian] http://payeshjournal.ir/article-1-279-en.html

[14] Khodabakhshi-koolaee A. The comparison of health literacy and lifestyle among retired and homemaker older adults' women. J Health Lit. 2016; 1(3):155-63. [In Persian] http://literacy.ihepsa.ir/article-1-46-en.html
[15] Reisi M, Javadzade SH, Babaei Heydarabadi A, Mostafavi F, Tavassoli $E$, Sharifirad Gh. Health literacy and health promoting behaviors among older adults. J Health Syst Res. 2013; 9(8):82736. [DOI:10.1007/s10903-012-9666-7] [PMID] [PMCID]

[16] Hosieni F, Mirzaei T, Ravari A, Akbary A. The relationship between health literacy and quality of life in retirement Rafsanjan University of Medical Sciences. J Health Lit. 2016; 1(2):929. [In Persian] http://literacy.ihepsa.ir/article-1-32-en.htm

[17] Baljani E, Rahimi JH, Amanpour E, Salimi S, Parkhashjoo M Effects of a nursing intervention on improving self-efficacy and reducing cardiovascular risk factors in patients with cardiovascular diseases. Hayat. 2011; 17(1):45-54. http://hayat.tums. ac.ir/article-1-70-en.html

[18] Naseh L, Shaikhy RA, Rafiei H. General Self-efficacy and associated factors among elderly residents of nursing home. J Holistic Nurs Midwifery. 2014; 26(80):90-7. https://www.sid. ir/en/journal/ViewPaper.aspx?id=510680

[19] Osborn CY, Paasche-Orlow MK, Bailey SC, Wolf MS. The mechanisms linking health literacy to behavior and health status. Am J Health Behav. 2011; 35(1):118-28. [DOI:10.5993/ AJHB.35.1.11] [PMID] [PMCID]

[20] Lee EH, Lee YW, Moon SH. A structural equation mode linking health literacy to self-efficacy, self-care activities, and health-related quality of life in patients with type 2 diabetes. Asian Nurs Res (Korean Soc Nurs Sci). 2016; 10(1):82-7. [DOI:10.1016/j.anr.2016.01.005] [PMID]

[21] Dominick GM, Dunsiger SI, Pekmezi DW, Marcus BH. Health literacy predicts change in physical activity self-efficacy among sedentary Latins. J Immigr Minor Health. 2013; 15(3):533-9. [DOI:10.1007/s10903-012-9666-7] [PMID] [PMCID]

[22] Peyman N, Abdollahi M. The relationship between health literacy and self-efficacy physical activity in postpartum women. J Health Lit. 2016; 1(1):5-12. [In Persian] http://literacy.ihepsa. ir/article-1-23-en.htm

[23] Rafiezadeh Gharrehtapeh Sh, Tabarsy B, Hassanjani S, Razavi $M$, Amjadi M, Hojjati H. Relationship between the Health Literacy with selfefficacy of the diabetic patient's type 2 referred to Gorgan city clinic in 2014. J Diabetes Nurs. 2015; 3(2)3:30-42. https://www.sid.ir/en/Journal/ViewPaper.aspx?ID=506587 
This Page Intentionally Left Blank 